\title{
Erratum: Photoconductivity in CdSe quantum dot solids [Phys. Rev. B 62, 2669 (2000)]
}

C. A. Leatherdale, C. R. Kagan, N. Y. Morgan, S. A. Empedocles, M. A. Kastner, and M. G. Bawendi (Published 22 December 2000)

DOI: 10.1103/PhysRevB.63.039901

PACS number(s): 73.61.- r, 73.50.-h, 99.10.+g

The last sentence of the abstract should read, "We show that the charge generation efficiency decreases with increasing temperature as nonradiative and radiative recombination pathways increasingly compete with charge separation.'

On page 2678, Eqs. (A2a) and (A2b) should read

$$
\begin{gathered}
E_{1}=\frac{-1.79 q^{2}}{4 \pi \varepsilon_{0} \varepsilon_{\mathrm{QD}} R}+\frac{q^{2}}{4 \pi \varepsilon_{0} R}\left(\frac{1}{\varepsilon_{\mathrm{QD}}}-\frac{1}{\varepsilon_{\mathrm{ave}}}\right)+2 \frac{q^{2}}{8 \pi \varepsilon_{0} \varepsilon_{\mathrm{QD}} R} S_{s}, \\
E_{2}=\frac{-q^{2}}{4 \pi \varepsilon_{0} \varepsilon_{\mathrm{ave}}(2 R+d)}+2 \frac{q^{2}}{8 \pi \varepsilon_{0} \varepsilon_{\mathrm{QD}} R} S_{s} .
\end{gathered}
$$

These corrections do not affect either the results or conclusions of the paper. 